\title{
Avaliação nutricional de crianças e adolescentes institucionalizados
}

\section{Nutritional assessment of institutionalized children and adolescents}

\author{
Veridiana Silva Corvelho' ${ }^{1}$, Bianca Inês Etges ${ }^{1}$ \\ 1 - Universidade de Santa Cruz do Sul - UNISC, Santa Cruz do Sul, RS, Brasil.
}

bianca@unisc.br

\begin{abstract}
RESUMO
Objetivo: avaliar o estado nutricional das crianças e adolescentes e relacionar com tempo de permanência na instituição COPAME, no município de Santa Cruz do Sul- RS. Método: trata-se de um estudo transversal, retrospectivo, de caráter quantitativo realizado em uma instituição de acolhimento para crianças em risco social. A amostra foi constituída por conveniência, com dados de prontuários de 31 crianças e adolescentes de 0 a 15 anos, abrigados na instituição COPAME, no período de julho de 2017 a setembro de 2018. A análise estatística foi realizada no programa Statistical Package for Social Sciences, versão 20.0. Primeiramente realizou-se uma análise descritiva a partir dos cálculos de média, desvios padrão e frequências. Utilizou-se o teste de Mann-Whitney ou teste T de Student para comparar os resultados médios da variável idade, peso e altura, o Qui-quadrado para verificar possíveis mudanças no estado nutricional e no consumo alimentar dos escolares antes e após o acolhimento e para a comparação das variáveis estado nutricional (antes e após o acolhimento) com sexo e motivo do abandono, considerando-se significativo um valor de $\mathrm{p}<0,05$. Resultados: a amostra estudada atendendo os critérios de inclusão e exclusão totalizou 31 crianças e adolescentes. Foi composta por $64,51 \%(n=20)$ crianças menores de 5 anos e $35,49 \%(n=11)$ maiores de 5 anos de idade. Em relação ao sexo, observou-se maior proporção de meninos $21(67,7 \%)$. O principal motivo que levou as crianças ao acolhimento foi negligência $(80,6 \%, n=25)$. A média de tempo de abrigamento das crianças foi de 6,90土5,93 meses. A maioria das crianças e adolescentes encontravam-se em eutrofia em todos os índices antropométricos avaliados no momento do acolhimento. A análise da relação entre sexo e motivo do acolhimento com os indicadores antropométricos utilizados para classificação

Palavras-chave: do estado nutricional não mostrou relação significativa em nenhuma das variáveis analisadas. Conclusão: ao analisar os dados antropométricos dos dois grupos etários estudados, 0 a 5 anos e 5 a 15 anos, acolhidas durante o período do estudo pode-se observar que houve uma piora do estado nutricional em todas as variáveis estudadas, em relação ao momento e após o abrigamento, uma parte das crianças apresentou o crescimento insatisfatório comparando com os padrões adotados pelo Mistério da Saúde.
\end{abstract}

Crianças; Adolescentes; Abrigamento; Estado

Nutricional.

\section{ABSTRACT}

Objective: to evaluate the nutritional status of children and adolescents and to relate them to length of stay at the COPAME institution, in the city of Santa Cruz do Sul, RS. Method: this is a cross-sectional, retrospective, quantitative study conducted in a host institution for children at social risk. The sample consisted of convenience, with records of 31 children and adolescents aged 0 to 15 years, housed at the COPAME institution, from July 2017 to September 2018. Statistical analysis was performed using the Statistical Package for Social Sciences, version 20.0. First, a descriptive analysis was performed based on the calculations of means, standard deviations and frequencies. The Mann-Whitney test or Student's t-test was used to compare the average results of the variable age, weight and height, the Chi-square to verify possible changes in the nutritional status and food consumption of students before and after admission and for the comparison of the variables nutritional status (before and after reception) with sex and reason for abandonment, considering a value of $\mathrm{p}<0.05$ as significant. Results: the sample studied meeting the inclusion and exclusion criteria totaled 31 children and adolescents. It was composed of $64.51 \%(\mathrm{n}=20)$ children under 5 years old and $35.49 \%(\mathrm{n}=11)$ older than 5 years old. In relation to sex, a higher proportion of boys were observed (21.7\%). The main reason that led the children to the institution was negligence $(80.6 \%, \mathrm{n}=25)$. The average shelter time of the children during the data collection period was $6.90 \pm 5.93$. The majority of the children and adolescents were in eutrophy in all the anthropometric indices evaluated at the time of arrival. The analysis of the relationship between sex and the reason for the admission of children and adolescents from 5 to 15 years old with the anthropometric indicators used to classify
Keywords: ${ }^{\text {the nutritional status did not show a significant relationship in any of the analyzed variables. Conclusion: when }}$ analyzing the anthropometric data of the two age groups studied, 0 to 5 years and 5 to 15 years, received during
Children; Adolescents; the study period, it can be observed that there was a worsening of nutritional status in all variables studied, in Shelter; Nutritional relation to the moment and after the shelter, a part of the children showed an unsatisfactory growth compared to Status. the standards adopted by the Ministry of Health. 


\section{INTRODUÇÃO}

Cerca de 30 mil crianças e adolescentes vivem em instituições de acolhimento no Brasil, devido principalmente a baixas condições socioeconômicas das famílias, ${ }^{1}$ o que muitas vezes acarreta prejuízo ao seu estado nutricional, observado principalmente nas crianças menores de cinco anos, pois o cuidado nesta fase é essencial. ${ }^{2} \mathrm{O}$ estado nutricional da criança reflete em suas condições de vida, pois o crescimento é sensível a diversos fatores. ${ }^{3}$

Os efeitos gerados pela ausência ou fragilidade dos vínculos familiares já estabelecidos podem ser agravados pela permanência prolongada em ambiente institucional. Ou seja, o efeito dessa vivência sobre a formação da identidade e o sentimento de insegurança emocional pode deixar a criança e o adolescente ainda mais vulneráveis a doenças físicas e psíquicas graves e/ou crônicas, hospitalizações prolongadas, dificuldades de relacionamento nos grupos de convívio social, entre outros danos. ${ }^{4}$

$\mathrm{O}$ acolhimento institucional, segundo descrito no Estatuto da Criança e do Adolescente (ECA), ${ }^{5}$ é uma medida de proteção de caráter provisório e excepcional (art. 101, § $1^{\circ}$ ), utilizada para crianças e adolescentes em situação de risco. Essa medida deve priorizar o fortalecimento dos laços familiares e comunitários, visando à reinserção da criança ou do adolescente em sua família natural ou extensa e, caso a reinserção não seja possível, sua colocação em família substituta. ${ }^{5}$ As instituições de acolhimento teriam, portanto, como um de seus principais objetivos, o autoconhecimento e a reconstrução das histórias de vida da população a que atendem. ${ }^{6}$

$\mathrm{O}$ acolhimento institucional, anteriormente denominado abrigamento em entidade, é uma das medidas de proteção previstas pela Lei Federal $\mathrm{n}^{\circ}$ 8069/1990-ECA aplicável a crianças e adolescentes, sempre que os direitos reconhecidos naquela lei forem ameaçados ou violados. ${ }^{5} \mathrm{~A}$ institucionalização é uma prática muito antiga de proteção de crianças e adolescentes. A partir de 1990, mais precisamente no Brasil, que se cria uma nova posição internacional oposta à institucionalização das crianças e adolescentes, reforçada pela criação do ECA, juntamente com a Convenção das Nações Unidas pelos Direitos da Criança. Neste mesmo período, aconteceram mudanças importantes em relação às crianças e adolescentes na sociedade, sendo que nessa fase teve grande marco em relação aos direitos dos mesmos. ${ }^{5}$ Foi a partir da vigência do ECA que começou a ser dado um olhar de prioridade às crianças e adolescentes, transformando-os em sujeitos de proteção e direitos. $^{7}$

O estado nutricional de crianças e adolescentes que se encontram institucionalizadas deve ser monitorado constantemente através da avaliação antropométrica, que consiste em um método simples e de baixo custo. ${ }^{8} \mathrm{O}$ método de avaliação do estado nutricional está diretamente relacionado com o objetivo da avaliação, qual seja: diagnosticar a proporção dos problemas nutricionais, identificar e analisar os seus determinantes para o estabelecimento de medidas de intervenção adequadas. ${ }^{8}$

Poucos estudos documentam a condição nutricional da criança na admissão nessas instituições, bem como os efeitos da institucionalização no seu desenvolvimento físico. Sendo assim, o presente estudo teve por objetivo avaliar o estado nutricional das crianças e adolescentes e relacionar com tempo de permanência na instituição COPAME, no município de Santa Cruz do Sul- RS.

\section{MÉTODO}

Trata-se de um estudo transversal, retrospectivo, de caráter quantitativo realizado em uma instituição de acolhimento para crianças em risco social, localizada na cidade de Santa Cruz do Sul - RS. A amostra foi constituída por conveniência, de dados de prontuários de 34 crianças e adolescentes de 0 a 15 anos, abrigados na instituição COPAME, no período de julho de 2017 a setembro de 2018. Adotaram-se como critérios de exclusão prematuridade, baixo peso ao nascer, síndrome genética ou doença crônica grave que pudessem levar ao déficit nutricional, paralisia cerebral, desconhecimento da data de nascimento da criança, prontuários com dados necessários incompletos. Atenderam a esses critérios 31 crianças e adolescentes.

Este estudo é resultado de um levantamento sobre o estado nutricional no momento da admissão e após o período de 1,2 anos ou desacolhimento. A estatura e o peso das crianças são aferidos pela nutricionista da entidade no momento da admissão, e repetido a cada 3 meses. Essa atividade faz parte da rotina da nutricionista.

A Associação Comunitária Pró-amparo ao Menor (COPAME) $)^{9}$ é uma instituição que atende crianças de até 12 anos de idade em regime de abrigo, 
diuturnamente, sendo mantida financeiramente por doações de pessoas físicas e jurídicas. Contribuições dos associados e convênios firmados com prefeituras da região. A COPAME é uma instituição que acolhe temporariamente crianças em situação de risco social ou abandono, promovendo sua proteção integral até a decisão judicial sobre seu destino, reintegração familiar ou sua adoção.

As variáveis estudadas foram sexo, idade, peso, estatura e motivo do abrigamento da criança na instituição; e os dados foram obtidos através dos formulários individuais das crianças. Para identificação dos motivos do acolhimento utilizou-se a primeira razão declarada no prontuário no momento do acolhimento na instituição ou o motivo principal que gerou o encaminhamento.

Para o diagnóstico nutricional, adotaram-se os seguintes índices: peso por idade $(\mathrm{P} / \mathrm{I})$, peso por estatura $(\mathrm{P} / \mathrm{E})$, estatura por idade $(\mathrm{E} / \mathrm{I})$ e índice de massa corporal $(\mathrm{kg} / \mathrm{m} 2)$ por idade (IMC/I), expressos em EscoreZ, considerando-se os pontos de corte da Organização Mundial da Saúde ${ }^{10}$, adotou-se a recomendação do Sistema de Vigilância Alimentar e Nutricional (SISVAN 2011), que define para os índices P/E e IMC/I: desnutrição (magreza e magreza acentuada), quando escore- $\mathrm{z}<-2$; eutrofia, quando Escore $Z$ for $\geq-2$ e $\leq+2$ e excesso de peso (sobrepeso e obesidade), quando Escore $Z>+2$. Para o índice E/I: baixa estatura, quando $<$ Escore $Z-2$; estatura adequada, quando Escore $Z \geq-2$. E para o índice $P / I$ : baixo peso, quando Escore $Z<-2$; peso adequado, quando Escore $Z$ for $\geq-2$ e $\leq+2$; e peso elevado, quando Escore $Z$ for $\geq+2$.

O IMC/I expressa a relação entre o peso da criança e o quadrado da estatura. Utilizado principalmente para identificar déficit nutricional e o excesso de peso entre crianças e adolescentes, tem a vantagem de ser um índice empregado em outras fases da vida.

A E/I é o índice que melhor aponta o efeito cumulativo de situações adversas sobre o crescimento da criança e do adolescente (valores abaixo do esperado indicam déficit de longa duração), sendo considerado o indicador mais sensível para aferir a qualidade de vida de uma população. ${ }^{11-13}$

A análise estatística foi realizada no programa Statistical Package for Social Sciences (SPSS) versão 20.0 (Chicago, IL). Primeiramente realizouse uma análise descritiva a partir dos cálculos de média, desvios padrão e frequências. Utilizou-se o teste de Mann-Whitney ou teste T de Student para comparar os resultados médios da variável idade, peso e altura, o Qui-quadrado para verificar possíveis mudanças no estado nutricional e no consumo alimentar dos escolares antes e após o acolhimento e para a comparação das variáveis estado nutricional (antes e após o acolhimento) com sexo e motivo do abandono, considerando-se significativo um valor de $p<0,05$. Essa pesquisa foi realizada após aprovação do Comitê de Ética da Universidade de Santa Cruz do Sul - RS, CAE 93325618.0.0000.5343. Parecer número: 2.809.259.

\section{RESULTADOS}

Das 34 crianças e adolescentes de 0 a 15 anos admitidas na instituição, entre julho de 2017 e setembro de 2018, 3 foram excluídas. Uma pela impossibilidade de aferição das medidas antropométricas no momento da admissão, 1 por estar internada por motivos de saúde e não possuir suas medidas antropométricas no prontuário e 1 por motivo de óbito.

A amostra estudada foi constituída de 31 crianças e adolescentes, composta por $64,51 \%(n=20)$ menores de 5 anos e 35,49\% ( $n=11)$ maiores de 5 anos de idade. Em relação ao sexo observou-se maior proporção de meninos $(67,7 \%, n=21)$. O principal motivo que levou as crianças ao acolhimento foi negligência $(80,6 \%, n=25)$. A média de tempo de abrigamento das crianças durante o período da coleta de dados foi de 6,90土5,93 meses (Tabela 1).

Tabela 1 - Descrição da amostra crianças e adolescentes $0-15$ anos $(n=31)$.

\begin{tabular}{lcc}
\hline Variável & $\begin{array}{c}\text { Frequência } \\
\mathbf{n}(\%)\end{array}$ & $\begin{array}{c}\text { Média } \pm \text { Desvio } \\
\text { padrão }\end{array}$ \\
\hline Sexo & $10(32,3 \%)$ & \\
Feminino & $21(67,7 \%)$ & \\
Masculino & $4(12,9 \%)$ & \\
Motivo do acolhimento & $2(6,5 \%)$ & \\
Abandono & $25(80,6 \%)$ & \\
Abuso & & $6,90 \pm 5,93$ \\
Negligencia & & \\
Tempo de acolhimento & & \\
(meses) & & \\
\hline
\end{tabular}

Das 31 crianças acolhidas durante o período da coleta de dados $67,74 \%(\mathrm{n}=21)$ permaneciam na instituição ao final desse período.

As crianças menores de 5 anos totalizaram 20, também com predominância do sexo masculino $(70,0 \%, n=14)$. Com relação aos motivos do acolhimento, $80,0 \%,(n=16)$ tiveram como principal 
motivo a negligencia. A média de tempo de acolhimento das crianças durante o período da coleta de dados ( 1 ano e 2 meses), foi de 6,10 15,93 meses (Tabela 2).

Tabela 2 - Descrição da amostra de crianças de 0-5 anos de idade $(\mathrm{n}=20)$.

\begin{tabular}{lcc}
\hline Variável & $\begin{array}{c}\text { Frequência } \\
\mathbf{n}(\%)\end{array}$ & $\begin{array}{c}\text { Média } \pm \text { Desvio } \\
\text { padrão }\end{array}$ \\
\hline $\begin{array}{l}\text { Sexo } \\
\text { Feminino }\end{array}$ & $6(30,0 \%)$ & \\
$\begin{array}{l}\text { Masculino } \\
\text { Motivo do acolhimento }\end{array}$ & $14(70,0 \%)$ & \\
$\begin{array}{l}\text { Abandono } \\
\text { Negligencia } \\
\text { Tempo de acolhimento } \\
\text { (meses) }\end{array}$ & $4(10,0 \%)$ & \\
\hline
\end{tabular}

Ao analisar os índices antropométricos das crianças de $0-5$ anos $(n=20)$, verificou-se na variável $\mathrm{P} / \mathrm{I}(Z$-escore) no momento do abrigamento que $100 \%$ das crianças estavam eutróficas, e depois do acolhimento $25 \%$ destas crianças encontravamse em baixo peso para a idade $(\mathrm{p}<0,001)$; em relação a variável $\mathrm{P} / \mathrm{E}$ (Z-escore), $75 \%$ das crianças estavam em eutrofia no momento do abrigamento e após esse percentual diminuiu, passando para 55\% $(\mathrm{p}=0,010)$. A variável IMC/I (Z-escore) mostra uma variação maior, comparada com as demais variáveis, e também apresenta os menores percentuais de eutrofia $(30,0 \%)$,variando desde magreza acentuada $(15,0 \%)$, magreza $(20,0 \%)$ a risco de sobrepeso $(35,0 \%) \quad(p=0,001)$; já a variável $\mathrm{E} / \mathrm{I}$ (Z-escore) classifica a maioria das crianças $(95 \%)$ no momento do abrigamento e $(70 \%)$ após com estatura adequada para idade $(\mathrm{p}=<0,001)$. Pode-se observar uma piora do estado nutricional em todas as variáveis estudadas (Tabela 3).

Tabela 3 - Distribuição das crianças com 0-5 anos segundo sexo, idade, classificação do estado nutricional, antes e após o acolhimento $(\mathrm{n}=20)$.

\begin{tabular}{lccc}
\hline Variável & $\begin{array}{c}\text { Antes } \\
\mathbf{n}(\mathbf{\%})\end{array}$ & $\begin{array}{c}\text { Depois } \\
\mathbf{n}(\mathbf{\%})\end{array}$ & p-valor \\
\hline Idade (meses), média \pm desvio padrão & $13,48 \pm 15,53$ & $20,14 \pm 16,83$ & $<0,001$ \\
Peso (kg), média \pm desvio padrão & $8,67 \pm 4,80$ & $<, 12 \pm 4,70$ & 0,007 \\
Altura (m), média \pm desvio padrão & $0,71 \pm 0,19$ & $0,73 \pm 0,23$ & $<0,001$ \\
Peso/Idade(Z-escore) & & $5(25,0 \%)$ & $15(75,0 \%)$ \\
Baixo peso para a idade & $0(0,0 \%)$ & & \\
Peso adequado para a idade & $20(100,0 \%)$ & $3(15,0 \%)$ \\
Peso/Estatura (Z-escore) & & $11(55,0 \%)$ \\
Magreza & $1(5,0 \%)$ & $4(20,0 \%)$ \\
Eutrofia & $13(65,0 \%)$ & $2(10,0 \%)$ \\
Risco de sobrepeso & $6(30,0 \%)$ & \\
Sobrepeso & $0(0,0 \%)$ & $6(30,0 \%)$ \\
IMC/Idade (Z-escore) & & $0(0,0 \%)$ \\
Magreza acentuada & $3(15,0 \%)$ & $5(25,0 \%)$ \\
Magreza & $4(20,0 \%)$ & $7(35,0 \%)$ \\
Eutrofia & $6(30,0 \%)$ & $2(10,0)$ \\
Risco de sobrepeso & $7(35,0 \%)$ & $2(10,0 \%)$ \\
Sobrepeso & $0(0,0 \%)$ & $4(20,0 \%)$ \\
Estatura/Idade (Z-escore) & & $14(70,0 \%)$ \\
Muito baixa estatura para a idade & $0(0,0 \%)$ & $<, 001$ \\
Baixa estatura para a idade & $1(5,0 \%)$ & $<0,001$ \\
Estatura adequada para a idade & $19(95,0 \%)$ & \\
\hline
\end{tabular}

A relação entre sexo e motivo do acolhimento de crianças de 0-5 anos com os indicadores antropométricos utilizados para classificação do estado nutricional não mostrou relação significativa com as variáveis analisadas.

As crianças e adolescentes de 5 a 15 anos totalizaram 11, também com predominância do sexo masculino $(63,6 \%, n=7)$. Com relação aos motivos do acolhimento, $81,8, \%,(\mathrm{n}=9)$ tiveram como principal motivo a negligencia. A média de tempo de abrigamento das crianças durante o período da coleta de dados ( 1 ano e 2 meses), foi de 9,70 $\pm 5,27$ meses (Tabela 4). 
Tabela 4 - Descrição da amostra crianças e adolescentes 5-15 anos $(\mathrm{n}=11)$.

\begin{tabular}{lcc}
\hline Variável & $\begin{array}{c}\text { Frequência } \\
\mathbf{n}(\%)\end{array}$ & $\begin{array}{c}\text { Média } \pm \text { Desvio } \\
\text { padrão }\end{array}$ \\
\hline Sexo & $4(36,4 \%)$ & \\
$\begin{array}{l}\text { Feminino } \\
\text { Masculino }\end{array}$ & $7(63,6 \%)$ & \\
$\begin{array}{l}\text { Motivo do acolhimento } \\
\text { Abuso }\end{array}$ & $2(18,2 \%)$ & \\
$\begin{array}{l}\text { Negligencia } \\
\text { Tempo de acolhimento } \\
\text { (meses) }\end{array}$ & $9(81,8 \%)$ & \\
\hline
\end{tabular}

Das variáveis estudadas para crianças e adolescentes de 5 a 15 anos observa-se que no índice IMC/ I 45,5\% $(n=5)$ estavam em eutrofia no momento do abrigamento e depois do abrigamento apenas $9,1 \%(n=1)$ estava em eutrofia, aumentando os pecentuais de magreza e magreza acentuada. Já no índice $\mathrm{E} / \mathrm{I} 100,0 \%(\mathrm{n}=11)$ estavam com a estatura adequada para idade no momento do acolhimento e após $54,5 \%(\mathrm{n}=6)$ permaneceram em eutrofia, o restante apresentou baixa estatura para idade. Percebe-se também nas crianças e adolescentes uma piora do estado nutricional após o abrigamento com as variáveis estudadas (Tabela 5).

Tabela 5 - Distribuição das crianças e adolescentes com 5-15 anos segundo sexo, idade, classificação do estado nutricional, antes e após o abrigamento $(\mathrm{n}=11)$.

\begin{tabular}{lccc}
\hline Variável & $\begin{array}{c}\text { Antes } \\
\mathbf{n}(\mathbf{\%})\end{array}$ & $\begin{array}{c}\text { Depois } \\
\mathbf{n}(\mathbf{\%})\end{array}$ & p-valor \\
\hline Idade (anos), média \pm desvio padrão & $9,16 \pm 2,45$ & 0,834 \\
Peso (kg), média \pm desvio padrão & $33,86 \pm 11,86$ & $9,00 \pm 3,48$ & 0,005 \\
Altura (m), média \pm desvio padrão & $1,36 \pm 0,14$ & $36,27 \pm 11,94$ & 0,014 \\
IMC/Idade (Z-escore) & $1,39 \pm 0,14$ & 0,323 \\
Magreza acentuada & $1(9,1 \%)$ & & \\
Magreza & $2(18,2 \%)$ & $3(27,3 \%)$ & \\
Eutrofia & $5(45,5 \%)$ & $4(36,4 \%)$ \\
Sobrepeso & $2(18,2 \%)$ & $1(9,1 \%)$ \\
Obesidade grave & $1(9,1 \%)$ & $2(18,2 \%)$ \\
Estatura/Idade (Z-escore) & & $1(9,1 \%)$ \\
Baixa estatura para a idade & $0(0,0 \%)$ & \\
Estatura adequada para a idade & $11(100,0 \%)$ & $5(45,5 \%)$ \\
\hline
\end{tabular}

A análise da relação entre sexo e motivo do acolhimento de crianças e adolescentes de 5 a 15 anos com os indicadores antropométricos utilizados para classificação do estado nutricional não mostrou relação significativa em nenhuma das variáveis analisadas.

\section{DISCUSSÃO}

No presente estudo foi observado que o principal motivo que levou as crianças ao acolhimento foi negligência $(80,6 \%, n=25)$. Segundo Santos (2004) ${ }^{14}$, em Porto Alegre, uma pequena amostra da Fundação de Proteção Especial constata que 78\% dos ingressos foram pelo mesmo motivo.

A negligência representa uma omissão em termos de prover as necessidades físicas e emocionais de uma criança ou adolescente. ${ }^{7}$ Ela é configurada como uma falha dos pais (ou responsáveis) em termos de alimentar, de vestir adequadamente. E quando tal falha não é o resultado das condições de vida além do seu controle.
A maioria (67,7\%) das crianças e adolescentes acolhidos pela COPAME nesse período foi do sexo masculino independente da idade. Em pesquisa realizada pelo Instituto de Pesquisa Econômica Aplicada $^{15}$, que analisou 626 instituições de abrigo em todas as regiões brasileiras, a maioria das crianças abrigadas eram do sexo masculino, encontram-se após a primeira infância e a parcela mais significativa está nos abrigos há um período entre dois e cinco anos. Isto é, neste grupo etário, para cada menina abrigada existem dois meninos na mesma condição. Esses dados parecem indicar que as meninas conseguem deixar mais facilmente as instituições do que os meninos porque retornam mais rápido à convivência com a própria família de origem, ou porque encontram uma família substituta, ou, ainda, porque conseguem mais rápido meio para viabilizar a própria sobrevivência de forma autônoma e independente. ${ }^{15}$

Ao analisar os dados antropométricos dos dois grupos etários estudados, 0 a 5 anos e 5 a 15 anos, acolhidas durante o período do estudo pode-se observar que houve uma piora do estado nutricional 
em todas as variáveis estudadas, em relação ao momento e após o abrigamento, uma parte das crianças apresentou o crescimento insatisfatório comparando com os padrões adotados pelo Mistério da Saúde. ${ }^{16}$

Como esses dados estão relacionados ao período da pesquisa, que avalia os dados antropométricos do momento do acolhimento e períodos subsequentes, isto é, período recente de acolhimento, essa piora do estado nutricional pode estar relacionada a questões afetivas. Do ponto de vista do desenvolvimento global da criança, percebe-se que as relações afetivas entre mãe e bebê ganharam destaque e existem vários autores que se dedicaram a estudar essas relações. As primeiras pesquisas tiveram início após a Segunda Guerra Mundial, em que crianças órfãs ou abandonadas pelas mães foram objeto de estudo. Na época, verificouse que a ausência materna provocava distúrbios em diversas áreas da vida da criança. Trabalhando em um orfanato perto dos Estados Unidos, Spitz ${ }^{17}$ observou que os bebês que eram alimentados e vestidos, mas quase não recebiam afeto, nem eram segurados no colo ou embalados, apresentavam uma síndrome que ele denominou de hospitalismo. Eles apresentavam dificuldades no seu desenvolvimento físico, tinham o rosto vazio de expressão, tinham insônia, faltavalhes apetite, não ganhavam peso e, aos poucos, iam perdendo o interesse por se relacionar. Assim se afirmou que a ausência das relações objetais, causada pela carência afetiva, detém o desenvolvimento em todos os setores da personalidade.

A relação entre sexo e motivo do acolhimento das crianças independente da faixa etária com os indicadores antropométricos utilizados para classificação do estado nutricional não mostrou relação significativa em nenhuma das variáveis analisadas. Estudo realizado em Recife que avaliou o estado nutricional de 166 crianças menores de 60 meses, de ambos os sexos, de uma instituição de acolhimento, no momento da admissão também não encontrou relação entre o estado nutricional, segundo os índices $\mathrm{P} / \mathrm{I}$ e E/I, e o sexo, idade ou motivo do abrigamento. ${ }^{18}$

A avaliação nutricional das crianças de 0-5 anos $(n=20)$ mostrou a maioria das crianças em eutrofia nos índices analisados, com exceção da variável IMC/I (Z-escore) que apresenta os menores percentuais de eutrofia (30,0\%), variando desde magreza acentuada $(15,0 \%)$, magreza $(20,0 \%)$ a risco de sobrepeso $(35,0 \%)(p=0,001)$. O risco de sobrepeso em $35 \%$ das crianças avaliadas corrobora vários estudos realizados nos últimos anos, que demonstram a transição nutricional que vem ocorrendo no Brasil. De acordo com dados da Organização Mundial da Saúde (OMS), houve um aumento da obesidade infantil, sendo que $7,3 \%$ das crianças menores de cinco anos estão acima do peso. ${ }^{19}$

Estudo realizado em Fortaleza em 2011, com 44 crianças abrigadas, na faixa etária de 0 a 6 anos, abordando a relação peso/idade, percebeu-se que a maioria $(61,4 \%)$ estava na faixa de normalidade nutricional, seguida pelas crianças que estavam com peso baixo $(20,4 \%)$. Apenas $4,5 \%$ das crianças foram classificadas como de peso muito baixo e $2,3 \%$ foram incluídas na faixa de sobrepeso. ${ }^{20}$

Já a variável E/I (Z-escore) classifica a maioria das crianças (95\%) no momento do abrigamento e $(70 \%)$ após com estatura adequada para idade $(\mathrm{p}=<0,001)$. Vários inquéritos nacionais demonstram diminuição expressiva da prevalência da baixa estatura em crianças menores de cinco anos, entre 1974-1975 e 2006-2007. ${ }^{2} \mathrm{O}$ comprometimento do crescimento linear ocorre após longos períodos de ingestão nutricional inadequada, ${ }^{21}$ e sua prevalência é um indicador útil das condições de vida de uma população, ${ }^{2}$ o que pode sugerir que a amostra estudada estivesse vivendo em condições satisfatórias anteriormente à admissão na instituição.

Estudo $^{22}$ observou que $72,7 \%$ das crianças apresentaram altura dentro dos limites esperados. Entretanto, foi evidenciada estatura baixa para a idade em 27,3\% das crianças, a qual prevaleceu na fase pré-escolar. Com relação ao percentil peso/ estatura, constatou-se que grande parte das crianças encontrou-se na faixa de normalidade, totalizando $84 \%$. Contudo, $11,4 \%$ das crianças encontraram-se na faixa de sobrepeso e 4,6\% na faixa de desnutrição aguda.

A escassez de estudos semelhantes dificultou a comparação e a discussão dos resultados, o que mostra certa falta de interesse sobre o tema pela comunidade científica. Somente um acompanhamento de forma contínua do crescimento e desenvolvimento de crianças e adolescentes institucionalizados poderá fornecer dados reais da situação nutricional dessa população.

Poucos estudos documentam a condição nutricional da criança na admissão nessas instituições, 
bem como os efeitos da institucionalização no seu desenvolvimento físico. ${ }^{20,22}$

Para enfrentar os problemas de abrigamento da infância, é importante que serviços de saúde a assistência públicos tenham uma visão integral sobre as famílias, pois crianças abrigadas estão entre os segmentos mais vulneráveis da população.

$\mathrm{O}$ ambiente institucional geralmente não oferece condições propícias para o pleno desenvolvimento de crianças, por uma série de razões, dentre as quais se podem citar a privação de contato íntimo com a família, elevado número de crianças por cuidadora, dificultando a estimulação do seu desenvolvimento e um elevado número de crianças por abrigo, acarretando em menor qualidade no cuidado e no estabelecimento de laços afetivos. ${ }^{23,24}$

\section{CONCLUSÃO}

Conclui-se que o principal motivo que levou as crianças à situação de acolhimento na instituição foi negligência e que os meninos foram mais prevelantes na amostra estudada de serem abrigados. Os dados antropométricos dos dois grupos etários estudados, 0 a 5 anos e 5 a 15 anos, acolhidos durante o período do estudo mostram que a maioria encontrava-se em eutrofia no momento do acolhimento e que houve uma piora do estado nutricional em todas as variáveis estudadas, em relação ao momento e após o abrigamento, em que uma parte das crianças e adolescentes apresentou o crescimento insatisfatório comparando com os padrões adotados pelo Mistério da Saúde.

\section{REFERÊNCIAS}

1. Brasil. Conselho Nacional do Ministério Público. Relatório da Infância e Juventude: resolução $n^{\circ}$ 71/2011: Um olhar mais atento aos serviços de acolhimento de crianças e adolescentes no País. Brasília; 2013.

2. Monteiro C, Benicio MHD, Conde WL, Konno SC, Lima ALL, Barros AJD, Victora CG. Desigualdades socioeconômicas na baixa estatura infantil: a experiência brasileira. Estudos Avançados 2013;27(78):35-49. doi: http://dx.doi.org/10.1590/ S0103-40142013000200004

3. Correia LL, Silva AC, Campos JS, Andrade FMO, Machado MMT, Lindsay AC, Leite AJM, Rocha HAL, Cunha AJLA. Prevalência e determinantes da desnutrição infantil no semiárido do Brasil. Rev Saude Publica 2014;48(1):19-28. doi: http:// dx.doi.org/10.1590/S0034-8910.2014048004828

4. Cavalcante LIC, Magalhães CMC, Pontes FAR. Processos de saúde-doença entre crianças institucionalizadas: Uma visão ecológica. Ciênc Saúde Coletiva 2009;14(2):614-625. doi:
http://dx.doi.org/10.1590/S1413-81232009000200030

5. Brasil. Lei $n^{0} 8.069$, de 13 de julho de 1990. Estatuto da criança e do adolescente. Brasília, DF; 1990.

6. Gulassa, MLCR. A fala dos abrigos. In: Baptista, M. V. (Org.). Abrigo: comunidade de acolhida e socio educação. São Paulo: Instituto Camargo Corrêa. 2006. 98 p.

7. Silva ML. Lei Nacional de adoção e acolhimento institucional: o ponto de vista de psicólogos e assistentes sociais [dissertação]. Santa Maria: Universidade Federal de Santa Maria; 2012.

8.Vasconcelos FAG. Avaliação nutricional de coletividades. Florianópolis: Ed. da UFSC; 1995. 154 p.

9. COPAME. Associação Comunitária Pró-amparo ao Menor. [documento na internet]. COPAME SCS: Associação Comunitária Pró Amparo do Menor [citado em 6 junho de 2018]. Disponível em: www.copame.org.br.

10. Araújo ACT, Campos JADB. Subsídios para Avaliação do Estado Nutricional de Crianças e Adolescentes por meio de Indicadores Antropométricos. Alimentos e nutrição [periódico online]. 2008 [capturado 2018 jan 23];19(2):219-25. Disponível em: https://biblat.unam.mx/pt/revista/alimentos-e-nutricao/ articulo/subsidios-para-a-avaliacao-do-estado-nutricionalde-criancas-e-adolescentes-por-meio-de-indicadoresantropometricos

11. Sigulem DM, Devincenzi UM, Lessa AC. Diagnóstico do estado nutricional da criança e do adolescente. J Pediatr 2000;76(Suppl 3):275S-84.

12. Duarte AC, Castellani FR. Semiologia nutricional. Rio de Janeiro: Axcel Books do Brasil; 2002. 128 p.

13. Sociedade Brasileira De Pediatria. Avaliação nutricional da criança e do adolescente: Manual de Orientação. São Paulo: Departamento de Nutrologia; 2009.

14. Santos, MI. Mapeando a realidade de um trabalho voluntário: levantamento estatístico. In: Azambuja MRF, Silveira MV, Bruno DD (Orgs). Infância em família: um compromisso de todos. Porto Alegre: Instituto Brasileiro de Direito de Família; 2004. P. 17-28.

15. Silva ERA. O direito à convivência familiar e comunitária: os abrigos para crianças e adolescentes no Brasil. Brasília: IPEA/ CONANDA; 2004.

16. World Health Organization - WHO. Child Growth Standards: Methods and development: Length/heightfor-age, weight-forage, weight-for-length, weight-forheight and body mass indexfor-age. Geneva; 2006.

17. Spitz, R. El primer ano Del vida Del niño. Madrid: Aguilar. 1958. $75 \mathrm{p}$.

18. Silveira AC, Maniçoba SC, Costa F, Pinho CPS, Santana SCS. Condição nutricional de crianças admitidas em uma instituição de acolhimento. Rev Bras Prom Saúde 2016; 29(1):5-13.

19. Organização das Nações Unidas para Alimentação e Agricultura (FAO). Aumentam sobrepeso e obesidade no Brasil, aponta relatório de FAO e OPAS [documento na internet]. Brasília: Casa ONU Brasil; 2017. [citado em 22 de maio de 2019]. Disponível em: https://nacoesunidas.org/aumentamsobrepeso-e-obesidade-no-brasil-aponta-relatorio-de-fao-eopas/

20. Torres CA, Santana JS Neto, Silva MMA, Rossi TRM, Souza LMF, Rocha NSPD. Crianças em passagem: um caminho para a cidadania? Rev Bras Med Fam Comunidade 2008;3(12):282-9. 21. Programa das Nações Unidas para o Desenvolvimento 
(PNUD), Instituto de Pesquisa Econômica Aplicada (IPEA). O índice de desenvolvimento Humano Municipal Brasileiro: atlas do desenvolvimento humano no Brasil [documento na internet]. Brasil: Atlas do Desenvolvimento Humano no Brasil; 2013 [citado em 2020 abril de 2017]. Disponível em: http:// www. atlasbrasil.org.br/2013/data/rawData/publicacao_ atlas municipal.pdf

22. Chaves, CMP, Lima FET, Mendoça LBA, Custódio IL, Matias EO. Avaliação do crescimento e desenvolvimento de crianças institucionalizadas. Ver Bras Enferm 2013;66(5):66874. doi: http://dx.doi.org/10.1590/S0034-71672013000500005

23. Lima AKP, Lima AO. Perfil do desenvolvimento neuropsicomotor e aspectos familiares de crianças institucionalizadas na cidade do Recife. Rev CES Psicol 2012;5(1):11-25. doi: http://dx.doi.org/10.21615/2051

24. Freitas HMB, Backes DS, Pereira ADA, Ferreira CLL, Marchiori MRC, Souza MHT, Erdmann AL. Significados que os profissionais de enfermagem atribuem ao cuidado de crianças institucionalizadas com AIDS. Texto \& Contexto Enferm 2010;19(3):511-7. doi: http://dx.doi.org/10.1590/S010407072010000300013

Como citar: CORVELHO, Veridiana Silva; ETGES, Bianca Inês. Avaliação nutricional de crianças e adolescentes institucionalizados. Revista Interdisciplinar de Promoção da Saúde, Santa Cruz do Sul, v. 2, n. 4, out. 2019. ISSN 2595-3664. Disponível em: <https://online.unisc.br/seer/index.php/ripsunisc/article/view/14838>. Acesso em: 01 out. 2019. doi:https://doi.org/10.17058/ rips.v2i4. 14838 